\title{
Influence of Immune Status on the Airborne Colonization of Piglets with Methicillin-Resistant Staphylococcus aureus (MRSA) Clonal Complex (CC) 398
}

\author{
Kerstin Rosen $^{1 *}$, Friederike Ebner ${ }^{2}$, Stefanie Schmidt ${ }^{2}$, Susanne Hartmann ${ }^{2}$, Roswitha Merle ${ }^{3}$, \\ Anika Friese $^{1}$ and Uwe Roesler ${ }^{1}$ \\ ${ }^{I}$ Institute for Animal Hygiene and Environmental Health, Centre for Infection medicine, Department for Veterinary Medicine, Freie \\ Universitaet Berlin, Berlin, Germany \\ ${ }^{2}$ Institute of Immunology, Centre for Infection medicine, Department for Veterinary Medicine, Freie Universitaet Berlin, Berlin, Germany \\ ${ }^{3}$ Institute for Veterinary Epidemiology and Biostatistics, Freie Universitaet Berlin, Berlin, Germany
}

Received: 11 Nov 2019; accepted: 14 Dec 2019

\begin{abstract}
Colonized vertebrates including humans and pigs are to date the main reservoirs of livestock-associated Methicillin-resistant Staphylococcus aureus (LA-MRSA). Currently, the mechanisms underlying colonization of pigs are not fully understood.

We investigated the influence of piglet pre-immune status on airborne MRSA colonization. Three groups of MRSA-negative piglets were primed and exposed to airborne LA-MRSA $\left(10^{4}\right.$ colony forming units $\left.(\mathrm{cfu}) / \mathrm{m}^{3}\right)$ in an aerosol chamber for $24 \mathrm{~h}$. One group was treated intramuscularly with dexamethasone $(1 \mathrm{mg} / \mathrm{kg}$ body weight) to imitate weaning stress. The second group was exposed to bacterial endotoxin containing MRSA aerosol. Both conditions play a role in the development of multifactorial diseases and may promote MRSA colonization success. The third group served as control.

The piglets' MRSA status was monitored for 21 days via swab samples. At necropsy, specific tissues and organs were analyzed. Blood was collected to examine specific immunological parameters.

The duration of MRSA colonization was not extended in both treated groups compared to the control group, indicating the two immune-status influencing factors do not promote MRSA colonization. Blood sample analysis confirmed a mild dexamethasone-induced immune suppression and typical endotoxin-related changes in peripheral blood. Of note, the low-dose dexamethasone treatment showed a trend of increased MRSA clearance.
\end{abstract}

Keywords: livestock, ST398, aerosol chamber, swine, antibiotic resistance

\section{Introduction}

More than 10 years after the rise of livestock associated methicillin-resistant Staphylococcus aureus (LA-MRSA) in 2005, LA-MRSA is still a major public health concern. Although its primary reservoir is pig farming, the so-called LAMRSA has been repeatedly isolated from dogs, cats, and horses $[1,2]$. Given the high prevalence of LA-MRSA in pig farming, possible spread within and between farms, and its zoonotic potential, detailed knowledge about transmission is essential. In Germany, an MRSA prevalence between 52\% and $96 \%$ is reported in pig farming [3]. We recently identified the airborne MRSA concentration of $10^{6}$ colony forming units $(\mathrm{cfu}) / \mathrm{m}^{3}$ as an effective dose for airborne MRSA colonization of piglets [4]. Piglets exposed to this MRSA concentration for $24 \mathrm{~h}$ in an aerosol chamber were persistently colonized with MRSA. An airborne MRSA concentration of $10^{4} \mathrm{cfu} / \mathrm{m}^{3}$ resulted in transiently colonized animals. In contrast to the mean concentration of $10^{2} \mathrm{cfu} / \mathrm{m}^{3}$ found in the barn air of pigsties [5], the experimental dose required for a permanent MRSA colonization is relatively high. We assume that successful colonization and the high MRSA prevalence detected in pigsties are multifactorial. In this work, we focused on the

*Author for correspondence: Institute for Animal Hygiene and Environmenta Health, Robert-von-Ostertag-Str. 7-13, 14163, Berlin; E-mail: Kerstin. Rosen@fu-berlin.de; tierhygiene@vetmed.fu-berlin.de; Phone: +49 30838 60908; Fax: +49 30838451863. immunological state of piglets and its impact on MRSA colonization. In intensive livestock farming, many stress factors influence immunity and contribute to decreased resistance to pathogens. According to Amadori et al., common sources of chronic stress include poor or harmful climate conditions, pain, and increased infection pressure [6]. These result in an increased glucocorticoid secretion with decreased immunity, predisposing for the onset of diseases with opportunistic pathogenic micro-organisms [7]. Previous work has demonstrated that stress, especially after changing the environment often coincides with the exposure to new micro-organisms resulting in increased susceptibility to infection [8]. Following the pathogenesis of multifactorial diseases, we assumed that stress imitated by dexamethasone treatment would enhance MRSA colonization of piglets after experimental exposure. In addition, diseases of the respiratory tract are among the most common diseases of pigs in intensive pig farming. Air contaminants, especially endotoxins, are also potential underlying causes affecting the respiratory tract. As a part of the outer membrane of Gram-negative bacteria, endotoxin plays an indisputable role in the development of respiratory diseases. Holst et al. reported that airborne endotoxin affects the respiratory health of pigs due to inflammation [9]. According to Urbain et al., endotoxin facilitates the respiratory diseases in pigs due to its proinflammatory nature [10]. In guinea pigs, endothelial cell damage was observed when these animals were exposed for several weeks to airborne endotoxin [11].

This is an open-access article distributed under the terms of the Creative Commons Attribution-NonCommercial 4.0 International License (https://creativecommons.org/licenses/by-nc/4.0/), which permits unrestricted use, distribution, and reproduction in any medium for non-commercial purposes, provided the original author and source are credited, a link to the CC License is provided, and changes - if any - are indicated. 
We, therefore, addressed the relationship between airborne endotoxin exposure and sensitization of the piglets' airways for MRSA colonization. The overall aim of our study was to investigate if stress induced by dexamethasone and the presence of airborne endotoxin might act as contributory factors for airborne MRSA colonization of pigs and, therefore, contribute to the high MRSA prevalence observed in pigsties.

\section{Material and Methods}

Study Design. In our study, we investigated the effect of immunomodulating factors on MRSA colonization of piglets. We pre-treated groups of piglets $(n=9)$ with dexamethasone (dexamethasone treated group, DG) or included bacterial lipopolysaccharides (LPS) from E. coli O111:B4 (endotoxin treated group, EG) during MRSA exposure. Both groups were exposed to aerosolized MRSA for $24 \mathrm{~h}$ using an aerosol chamber. A control group $(\mathrm{CG})$ of 9 piglets $(n=9)$ was exposed to airborne MRSA alone as described previously [4]. All 3 groups were exposed to an airborne MRSA concentration of $10^{4} \mathrm{cfu} / \mathrm{m}^{3}$ as we previously demonstrated that this dose results in transient MRSA colonization of piglets [4]. Blood samples were taken to investigate the total leucocytes, including differential blood counts and the proliferation capacity of $\mathrm{CD} 4^{+} \mathrm{T}$ cells. To monitor the MRSA status of the piglets, 5 different swab samples (nasal, skin, pharyngeal, conjunctival, and rectal swab) were taken from each piglet 3 times a week for 21 days (observation period). At the end of the experiment, piglets were sacrificed by euthanasia to investigate the spread of MRSA into different tissues and organs. All samples were analyzed qualitatively and quantitatively for the presence of MRSA.

Aerosol Chamber. To expose piglets to MRSA, an aerosol chamber with a base area of $3.2 \mathrm{~m}^{2}$ and a volume of $7 \mathrm{~m}^{3}$ was used as described previously [4]. The MRSA suspension was prepared and transported by a perfusion pump with a rate of $9 \mathrm{~mL} / \mathrm{h}$ to an ultrasonic nebulizer (Broadband Ultrasonic Generator, Sono-Tek) that generated the aerosol. A ceiling ventilator dispersed the aerosol whose particle size distribution was measured by a spectrometer monitor (Grimm, model 1.109, GRIMM Aerosol Technik Ainring GmbH \& Co. KG, Germany). During exposure, the aerosol chamber was equipped with rubber mats covering half of the ground. The piglets were provided with feeding and water troughs and were allowed to move freely in the aerosol chamber.

During MRSA exposure, air samples were taken using an AGI-30 Impinger (Zinsser Analytic, Germany) to confirm the targeted MRSA concentration in the air and to investigate the aerosol distribution within the aerosol chamber. The impinger sampling positions were at 2 different heights (middle position $-0.8 \mathrm{~m}$ (MP) and high position - $1.6 \mathrm{~m}$ (HP)) and filled with $30 \mathrm{~mL}$ of phosphate-buffered saline (PBS) serving as sampling fluid.

Bacterial Strain and Preparation of MRSA Suspension. For our experiments, a MRSA strain (strain ID: BfR 08S00974, ITU 1179) of the sequence type (ST) 398 and the spa type t011 was obtained from the "Federal Institute for Risk Assessment" (BfR) and used as previously reported [4, 12]. Briefly, MRSA was streaked out on blood base agar plates (Blood Agar Base No. 2, Oxoid, Germany) and incubated for $8 \mathrm{~h}$. Then, the plates were suspended with PBS, adjusted to McFarland 0.5 and diluted to the defined MRSA concentration. The suspension was split into portions of $50 \mathrm{~mL}$ and stored on ice until aerosolization.

Animals and Animal Housing. The piglets arrived at our facilities at the age of approximately 3 weeks and were exposed to airborne MRSA $\left(10^{4} \mathrm{cfu} / \mathrm{m}^{3}\right) 7$ days later (day -1 ; Figure 1). For this study, 27 weaned, gender-mixed piglets were used. Two groups ( $n=9$, each) were used to investigate the effects of immunomodulatory factors on the MRSA colonization success. A third group $(n=9)$ served as control and was exposed to airborne MRSA without additional immunomodulating factors.

The piglets were housed at the research facility of the Centre for Infection Medicine of Freie Universitaet Berlin under a strict hygiene management. During the handling of the animals, protective clothes, snoods, and masks were used. The pig barn was cleaned daily with water. All persons in contact with the piglets, the pig barn itself, and the aerosol chamber were confirmed to be MRSA-negative before arrival of the piglets. During the experiment, the piglets' behavior and general condition were monitored daily. Internal temperature was measured 3 times per week and before taking the swab samples. Body weight development was monitored at least once weekly.

Dexamethasone Treatment. As presented in Figure 1, the piglets were treated with dexamethasone (dexamethasone $4 \mathrm{mg} / \mathrm{mL}$, Vetiquinol) on 9 consecutive days. The treatment started the day after arrival (day -6 ) and ended 2 days after airborne MRSA $\left(10^{4} \mathrm{cfu} / \mathrm{m}^{3}\right)$ exposure in the aerosol chamber (day 2), aiming an MRSA exposure under dexamethasone treatment. Dexamethasone was applied intramuscularly (i.m.) with a dosage of $1 \mathrm{mg} / \mathrm{kg}$ body weight. Prior to the dexamethasone application, each DG piglet was weighed daily to calculate the exact amount of dexamethasone applied.

Airborne Bacterial Endotoxin Treatment during MRSA Exposure. The second group (EG) was challenged simultaneously with bacterial endotoxin and airborne MRSA $\left(10^{4} \mathrm{cfu} / \mathrm{m}^{3}\right)$ for $24 \mathrm{~h}$. LPS from E. coli O111:B4 (Sigma Aldrich, Darmstadt, Germany) was added to the MRSA suspension used for aerosolization in the aerosol chamber. We chose an endotoxin concentration of $4 \mu \mathrm{g} / \mathrm{m}^{3}$ as this was the maximum concentration found in the air of pig barns by Zejda et al. [13]. To achieve the targeted endotoxin concentration in air, the required amount of the LPS-powder in the MRSA suspension for aerosolization was calculated. We dissolved $0.9 \mathrm{mg}$ of LPS per $\mathrm{mL}$ of bacterial suspension and vortexed the suspension gently.

Blood Samples. To investigate specific immunological parameters, blood samples of at least $5 \mathrm{~mL}$ were taken at different days prior to and after aerosol exposure, at day -6 , day -4 , day -1 , day 1 , day 3 , day 6 , day 13 , and day 21 from all piglets (Figure 1). The blood was taken from the vena cava cranialis with single-use needles $\left(\varnothing / L 0.90 \times 40\right.$, Sterican ${ }^{\circledR}$, B. Braun Melsungen AG, Germany) and VACUETTE blood tubes with lithium heparin (Greiner Bio-One, Germany). The first blood sample of the DG was taken prior to the first application of dexamethasone. Blood samples were examined by the Institute of Immunology (Freie Universitaet Berlin).

\section{Samples}

Aerosol Chamber. We used PBS-moistened cotton gauze to confirm the absence of MRSA on the floor (1 sample) and the chamber walls ( 2 different sampling locations) at a height of $1.5 \mathrm{~m}$ before starting the animal exposure in the aerosol chamber. For this purpose, an area of $900 \mathrm{~cm}^{2}$ was scrubbed.

During aerosolization, the airborne MRSA concentration was measured via impingement for $30 \mathrm{~min} 1 \mathrm{~h}, 9 \mathrm{~h}$, and $17 \mathrm{~h}$ after starting the MRSA exposure of the piglets to confirm the targeted bacterial load in the air.

After $24 \mathrm{~h}$ of MRSA exposure, the MRSA status of the aerosol chamber was investigated by sampling the same areas (wall and floor), using a PBS-moistened cotton gauze as described above. 


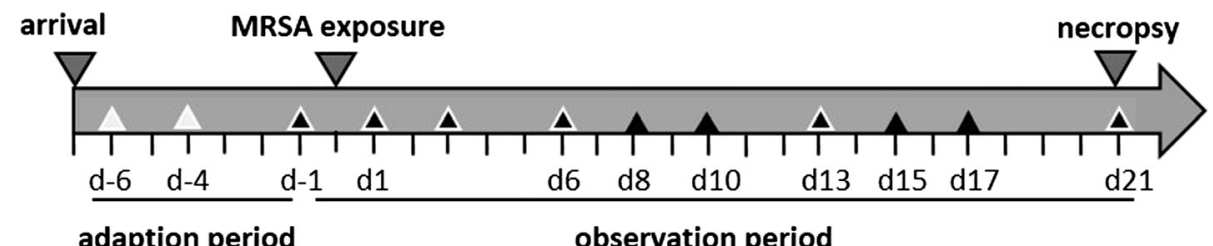

adaption period

observation period
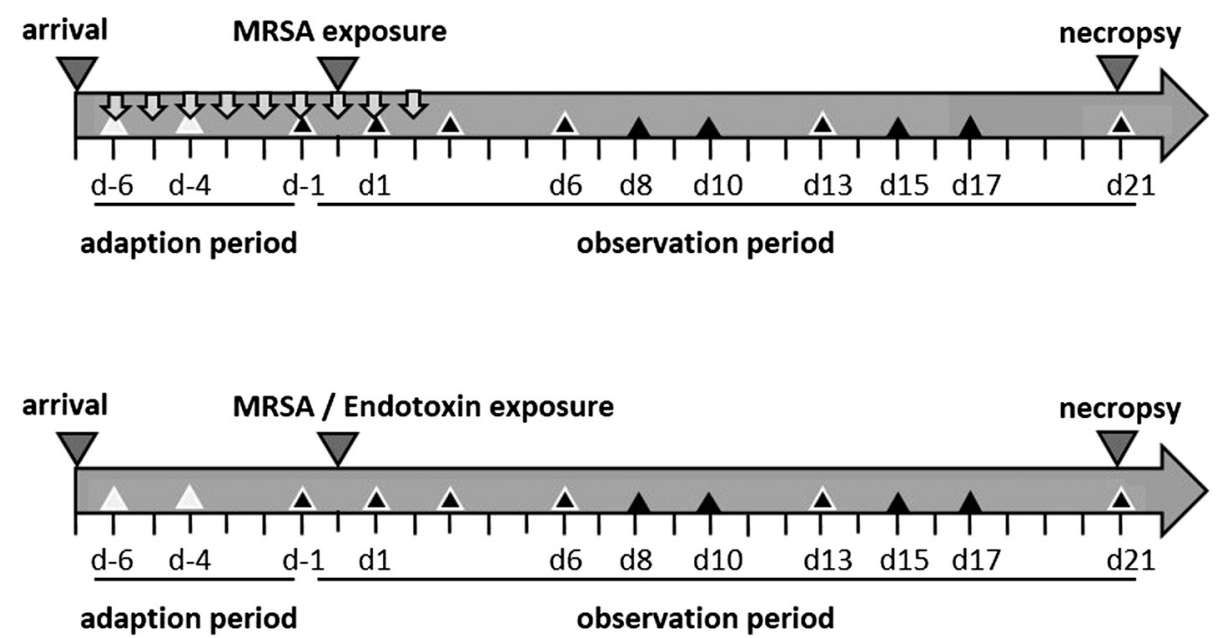

$\begin{array}{ll}\text { A. } & \text { swab samples } \\ \sqrt{\Delta} \text { Dexamethasone } \\ \text { A } & \text { swab + blood samples } \\ \nabla & \text { MRSA / Endotoxin exposure }\end{array}$

Figure 1. Study design

Animal Samples. To monitor the MRSA status of the piglets, nasal, pharyngeal, conjunctival, skin, and rectal swabs were taken directly before and after exposure (day -1 and day 1; Figure 1). As seen in Figure 1, the sampling was performed 3 times weekly during the ensuing observation period of 21 days as described previously [4]. After the observation period, necropsy was performed according to Szabó et al. and Rosen et al. to investigate the spread of MRSA into the tissues and organs $[4,12]$. We examined palatine tonsils, tracheal bifurcation, lung, spleen, and lymph nodes at various sites (ileocaecal, mandibular, and lung) qualitatively and quantitatively for the presence of MRSA.

Environmental Samples. To monitor environmental MRSA contamination, we sampled 5 different locations in the barn. Approximately $20 \mathrm{~cm}^{2}$ of the wall and the ground of the pig barn at 2 different locations were sampled. In addition, the feeding and water troughs, as well as the enrichment toy, were scrubbed at 1 position. Sampling proceeded within $2 \mathrm{~h}$ in the laboratory using PBS-moistened swabs with a diameter of $5 \mathrm{~mm}$ (Nerbe Plus GmbH, Winsen [Luhe], Germany).

Laboratory Analyses

Air Samples. After taking the air samples using impingement, the remaining PBS was measured, and a volume of $100 \mu \mathrm{L}$ of the sampling fluid was streaked out 3 times directly onto chromatic MRSA screen agar (CHROMagar MRSA; MAST Diagnostica $\mathrm{GmbH}$ ) and incubated aerobically at $37{ }^{\circ} \mathrm{C}$. After $24 \mathrm{~h}$, the MRSA concentration was determined by counting the typical MRSA colonies on the plate.

Swab Samples. All swabs were analyzed qualitatively for the presence of MRSA as described previously [4]. In brief, the swabs were extracted with PBS and vortexed. The fluid including the swab was transferred to a two-step-enrichment with Müller Hinton Broth (Oxoid, Germany) supplemented with $6.5 \% \mathrm{NaCl}(\mathrm{MHB}+)$ and Tryptone Soy Broth (Oxoid, Wesel, Germany) containing $75 \mathrm{mg} / \mathrm{L}$ aztreonam (Molekula $\mathrm{GmbH}$, Germany) and $3.5 \mathrm{mg} / \mathrm{L}$ cefoxitin (Fluka Analytical, Germany) (TSB + ). TSB + was streaked out onto chromatic MRSA screen agar and incubated overnight at $37{ }^{\circ} \mathrm{C}$. Suspicious colonies were confirmed using matrix-assisted laser desorption ionization-time of flight mass spectrometry (MALDI-TOF MS; MALDI Microflex LT and Biotyper database, Bruker Daltonics, Bremen, Germany).

Internal Organs. The tissues and organs of the dissected piglets were handled as described previously [4]. Briefly, the samples were decontaminated, cut into pieces, homogenized using a stomacher (stomacher 400 circulator; Seward Limited, West Sussex, United Kingdom) and enriched in the two-stepenrichment as mentioned above for the swab samples. A retained sample was stored at $4{ }^{\circ} \mathrm{C}$ and quantified in the case of positive results of the enrichment of the associated sample. Confirmation of MRSA suspected colonies was performed as described.

Spa Typing of MRSA Isolates. One isolate of every impinger sample for each group and 1 MRSA isolate of the last positive nasal swab of each animal were spa typed. The isolates were confirmed as spa type t011 by performing the PCR according to Kahl et al. [14]. LGC Genomics GmbH (Location) performed the sequencing. The sequences were analyzed using BioNumerics version 6.6.

PBMC Isolation and Proliferation Assay. Mononuclear cells from porcine peripheral blood (PBMC) were isolated by density centrifugation of whole blood diluted $1: 2$ in $0.9 \%$ 
$\mathrm{NaCl}$ using Pancoll solution (density $1.077 \mathrm{~g} / \mathrm{mL}$, PANBiotech). PBMC were stained with the proliferation marker carboxyfluorescein diacetate succinimidyl ester (CFSE, eBiosciences) at a concentration of $5 \mathrm{mM}$ for $5 \mathrm{~min}$ in the dark. CFSE-labeled porcine PBMCs were transferred to IMDM supplemented with $10 \%$ FCS and $1 \%$ penicillinstreptomycin (all PAN-Biotech, Aidenbach, Germany) and seeded into 96-well round bottom plates ( 2 Mio cells $/ 200 \mu \mathrm{L}$ ). Proliferation was induced by adding Concanavalin A (ConA, $2 \mu \mathrm{g} / \mathrm{mL}$; Sigma-Aldrich) and assessed after 5 days by comparing unstimulated and ConA-stimulated PBMC using flow cytometry.

For flow cytometry, cells were stained with the following antibodies specific to pig species: anti-CD4a-Pe-Cy7 (clone 412-4, IgG2b, BD Biosciences), anti-CD3ع-PerCP-Cy5.5 (clone BB23-8E6-8C8, IgG2a, BD Biosciences) and anti-CD8 $\alpha-$ AlexaFluor $^{\circledR} 647$ (clone 76-2-11, IgG2a, BD Biosciences). For dead cell exclusion, a fixable viability dye was used in eFluor $^{\circledR} 780$ (eBiosciences). Cells were acquired on BD FACS Canto II with BD FACS Diva software and analyzed using FlowJo v9 software (Tree Star) for proliferative capacity of $\mathrm{CD} 4+\mathrm{T}$ cells identified as liveCD ${ }^{+} \mathrm{CD} 4{ }^{+} \mathrm{CFSE}^{\text {low }}$.

Total Leucocytes and Differential Cell Counts. For total leucocyte counts, blood was treated with a red blood cell (RBC) lysis buffer $\left(150 \mathrm{mM} \mathrm{NH}{ }_{4} \mathrm{Cl}, 10 \mathrm{mM} \mathrm{KHCO}_{3}\right.$, and $0.1 \mathrm{mM} \mathrm{Na}{ }_{2}$ EDTA), and white blood cells (WBC) were counted using a Neubauer chamber (Marienfeld, Germany). To determine differential WBC counts, a blood smear was Romanowsky stained (DiffQuick, Labor + Technik, Eberhard Lehmann GmbH, Germany) and 200 cells were counted and classified. Percentages of lymphocytes, neutrophils, eosinophils, basophils, and monocytes were calculated. Absolute values were calculated by multiplying the total number of leucocytes with the percentage of each cell type.

Statistical Analysis. The software SPSS, version 24 (SPSS, Inc., Chicago, IL) was used to perform the statistical analysis. We used generalized regression models to estimate the effect of possible predisposing factors on the prevalence of MRSApositive individuals in population (logistic regression models) or on the number of log cfu per individual sample (linear regression models). Animal and type of swab sample were considered as random factors, while day of sampling was considered as repeated measurements in all models. The same analyses were also carried out, stratified in terms of the selected types of swab samples and the differences between 2 groups.

$P$ values $<0.05$ were regarded statistically significant. Model diagnostics included normality tests of residuals and visual inspection of homoscedasticity. The displayed results refer to the multivariable models described above.

For analyzing the blood data, statistical analyses were performed using ANOVA with repeated measurements. It was investigated if the percentage of the different cell types in blood differed between the groups "Dexamethasone", "Endotoxin", and "Control" in the course of time. Depending on the results of Mauchly's test of sphericity the parametric, the Greenhouse-Geisser or the Huynh-Feldt estimate was used to test differences between time. The Tukey test was used for multiple comparisons between the groups.
The proliferation data of $\mathrm{T}$ cells were analyzed by performing the $t$-test for paired samples, because we compared the animals' data of the "Dexamethasone" and "Control" group at 2 different time points.

Ethics. The animal study was approved by the German Animal Ethics Committee for the protection of animals of the Regional Office of Health and Social Affairs Berlin, Germany (Landesamt für Gesundheit und Soziales Berlin; approval number 0403/12). Piglets were cared for in accordance with the principles outlined in the European Convention for the Protection of Vertebrate Animals used for Experimental and other Scientific Purposes and in the German Animal Welfare Law.

\section{Results}

Airborne MRSA Concentration Measured during Piglets' Aerosol Exposure. The data for airborne MRSA concentration during exposure indicated the reliable reproducibility of the defined airborne MRSA concentration in the aerosol chamber (Table 1). The close values of minimal and maximal MRSA concentration in the air within each group demonstrated that MRSA was well distributed in the aerosol chamber air.

The Grimm aerosol spectrometer measured a particle size between $3.1 \mu \mathrm{m}$ and $3.7 \mu \mathrm{m}$ for all groups.

Prior to MRSA exposure, the aerosol chamber was tested MRSA-negative via sampling the floor and walls for all groups. After exposure, MRSA was detectable qualitatively on the floor and the walls of the aerosol chamber for all groups.

Clinical Symptoms. None of the animals showed any clinical signs during the experiment and the complete observation period. The body weight of the housed animals developed similarly to piglets of the same age.

General MRSA Colonization of the Pigs. Neither the DG nor the EG showed an extended MRSA colonization compared to the CG. The likelihood of animals being MRSApositive over the course of time was significantly higher ( $p \leq 0.001$, multivariable mixed logistic regression) for animals of the CG compared to the DG. Statistical analyses revealed no significant difference ( $p=0.103$, linear regression model) in the MRSA concentration between quantifiable swab samples of the DG and the CG over time. The probability of the animals to be MRSA-positive during the observation period was investigated using ANOVA with repeated measurements. No significant difference between the $\mathrm{CG}$ and EG $(p=0.145)$ could be shown. Similarly, the MRSA concentration in swab samples of the CG and EG did not differ statistically significantly $(p=0.130$, linear regression model).

Total MRSA-Positive Swab Samples over the Entire Observation Period. Figure 2 shows the number of MRSApositive swab samples per animal of all 3 groups for the respective sampling points during the entire observation period. In all groups, the number of MRSA-positive swab samples per animal was highest directly after exposure (day 1) and decreased over time. Like the CG and EG, all piglets $(n=9 / 9)$ of the DG showed MRSA-positive swabs directly after exposure (day 1) with a median of 3 positive swab

Table 1. MRSA concentration in the air in $\mathrm{cfu} / \mathrm{m}^{3}$ of the exposure of the control group (CG), dexamethasone-treated group (DG), and endotoxin-challenged group (EG). The data shown here are based on 3 measurements using 2 impingers (HP and MP) during the 24-h animal exposure

\begin{tabular}{lcccc}
\hline & & Control group $\left(3 \times 10^{4} \mathrm{cfu} / \mathrm{m}^{3}\right)$ & Dexamethasone group $\left(3 \times 10^{4} \mathrm{cfu} / \mathrm{m}^{3}\right)$ & Endotoxin group $\left(3 \times 10^{4} \mathrm{cfu} / \mathrm{m}^{3}\right)$ \\
\hline Animal & Mean & MRSA in air $\left(\mathrm{cfu} / \mathrm{m}^{3}\right)$ & MRSA in air $\left(\mathrm{cfu} / \mathrm{m}^{3}\right)$ & $2.1 \mathrm{E}+04$ \\
Exposure & Min. & $3.6 \mathrm{E}+04$ & $1.5 \mathrm{E}+04$ & $3.2 \mathrm{E}+04$ \\
& Max. & $1.6 \mathrm{E}+04$ & $2.7 \mathrm{E}+04$ & $2.5 \mathrm{E}+04$ \\
\hline
\end{tabular}


(A)

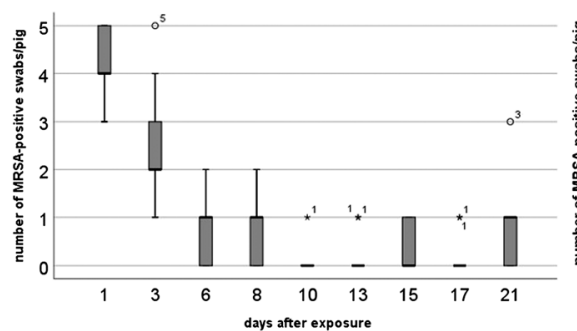

(B)

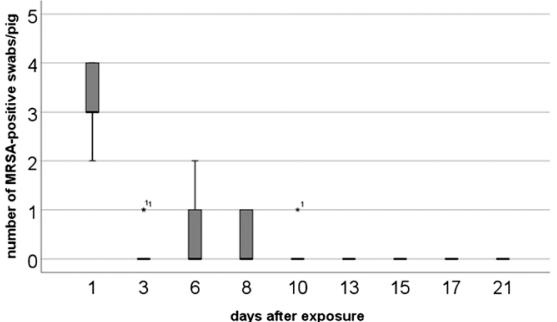

(C)

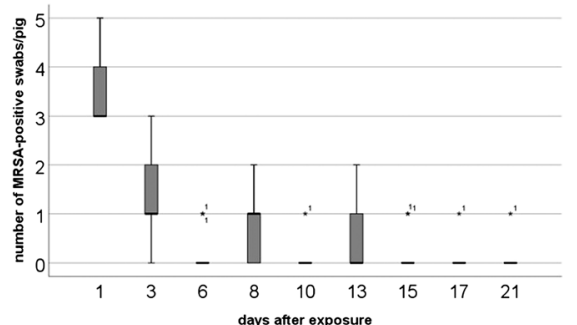

Figure 2. Mean count of MRSA-positive swabs per piglet of the control group (A), dexamethasone group (B), and endotoxin group (C) exposed to $10^{4} \mathrm{cfu} / \mathrm{m}^{3}$ MRSA in the air during the entire observation period over the observation period of 21 days. Boxes show the lower quartile, median, and the upper quartile. The ends of the whiskers show the lowest datum within 1.5 interquartile range of the lower quartile and the highest datum within 1.5 interquartile range of the lower quartile and the highest datum within 1.5 interquartile range of the upper quartile. Dots represent the outliners. Asterisk represents extreme values

samples per animal (Figure 2B). Most of them were nasal $(n=9 / 9)$, skin $(n=9 / 9)$, and rectal swabs $(n=7 / 9)$. For the further sampling points, $80 \%$ of all detected MRSA-positive samples of the DG were derived from skin swabs, whereas the last MRSA-positive swab sample was obtained at day 10 from 1 animal. In the DG, from day 3 to day $10,1(n=1 / 9)$ to 3 $(n=3 / 9)$ animals showed at least 1 MRSA-positive swab sample. In the EG, the number of MRSA-positive samples per animal decreased from a median of 3 positive swab samples per animal at day 1 to less than 1 MRSA-positive swab sample at the end of the observation period (Figure 2C). The number of MRSA-positive animals decreased from all piglets $(n=9 / 9)$ of the EG directly after exposure to $1(n=1 / 9)$ for the last sampling points (day 17 and day 21). According to Figure 2A, a median of 4 swab samples per piglet of the CG at day 1 was observed and decreased to less than 1 MRSApositive swab at day 21 , whereas $55 \%$ of the animals $(n=5 /$ 9) of this CG were still MRSA-positive at this time point.

The MRSA status of each animal over the course of time is depicted in the Supplementary Material (S1).

Nasal, Skin, and Pharyngeal Swabs. The stratified multivariable mixed logistic regression model for nasal swabs revealed a significantly higher probability for animals of the CG to have MRSA-positive nasal swabs $(p \leq 0.001)$ and skin swabs $(p \leq 0.001)$ during the experiment compared to the those of the DG. In contrast, there were no significant differences in the probability of having MRSA-positive nasal $(p=0.695)$ and skin swabs $(p=0.081)$ between the CG and EG group (stratified multivariable mixed logistic regression model for nasal and skin swabs). According to the pharyngeal swabs, there were no significant differences in the probability of the animals having MRSA-positive samples between all the 3 groups ( $p=0,550 \mathrm{DG}$ versus CG and $p=0,787 \mathrm{EG}$ versus $\mathrm{CG}$, stratified multivariable mixed logistic regression model for pharyngeal swabs).

As presented in Figure 3A and 3B, all animals of the DG showed MRSA-positive nasal and skin swabs directly the exposure (day 1). Although the nasal swabs remained MRSAnegative in the DG, MRSA-positive skin swabs were detectable until day 10 with a percentage between $11 \%(n=1 / 9)$ at day 3 and day 10 as well as $33 \%(n=3 / 9)$ at day 3 and day 6. As shown in Figure 3C, 2 animals $(n=2 / 9)$ of the DG showed MRSA-positive pharyngeal swabs directly after exposure (day 1). The last MRSA-positive swab of the pharynx was detected $(n=1 / 9)$ at day 3 .

All animals $(n=9 / 9)$ of the EG had MRSA-positive nasal and skin swabs at day 1 . Then, in both types of swab samples, the number of MRSA-positive swabs decreased, and MRSA was found only sporadically at different sampling points (Figure 3A and 3B). As seen in Figure 3C, 7 out of 9 pharyngeal swabs $(n=7 / 9)$ were MRSA-positive at day 1 for the EG. The MRSA detectability decreased at day 3, and MRSA was found again at day 15 only.

The results of CG were previously published [4]. In summary, almost all nasal, skin, and pharyngeal swabs of the CG were MRSA-positive directly after exposure (Figure 3A-3C). For all types of samples, the number of MRSA-positive swabs decreased over time.

Conjunctival and Rectal Swab. As presented in Figure 3D, the animals of the DG showed MRSA-positive conjunctivas on day $1(n=2 / 9)$ and day $6(n=1 / 9)$ only. The MRSApositive rectal swabs were observed exclusively in $77 \%$ $(n=7 / 9)$ of the DG's piglets directly after exposure (Figure $3 \mathrm{E}$ ).

The MRSA-positive conjunctival swabs of the EG were noted at day 1 and day 21 only (Figure 3D). There were only 2 animals in the EG showing MRSA-positive rectal swabs at day $1(n=2 / 9)$ and $1(n=1 / 9)$ animal at day 3 (Figure 3E).

More than half of the animals $(n=5 / 9)$ from the CG cohort showed MRSA-positive conjunctival swabs at day 1 (Figure 3D). Then, MRSA was detected sporadically. The MRSA-positive rectal swabs of the CG were observed in more than half of the animals $(n=5 / 9)$ at day 1 . Afterwards, MRSA was detectable sporadically only.

MRSA in the Experimental Environment of the Kept Animals. During the observation period, 5 different swab samples of the pig animal facility were taken to observe the MRSA status of the piglets' environment as a possible source for recolonization. The environmental swabs of the DG and EG groups were MRSA-positive directly after exposure (day 1) only. For the DG, MRSA was found on the wall, water, and feeding trough. For the EG, MRSA was also detectable on the ground floor. For the CG, with the exception of days 15 and 17 , at least 1 environmental swab was MRSA-positive over the entire observation period.

Internal Organs. MRSA was not found in tissues or organs of any group 21 days after MRSA exposure.

Spa Typing. The selected isolates were confirmed as spa type t011.

Immunological Parameters of the Blood Samples. Figure 4A shows the total leucocyte counts and differential white blood cell counts of the dexamethasone-treated animals prior to the first administration of dexamethasone (day -6) and $48 \mathrm{~h}$ after (day -4 ) compared to the CG. We observed a significant decrease in the total leucocyte count $(p=0.003$, 
(A)

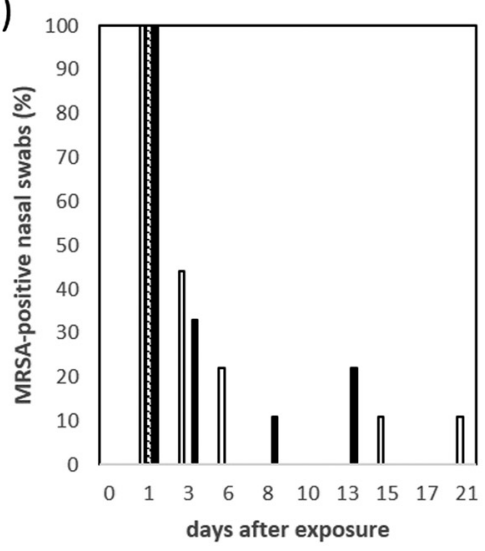

(D)
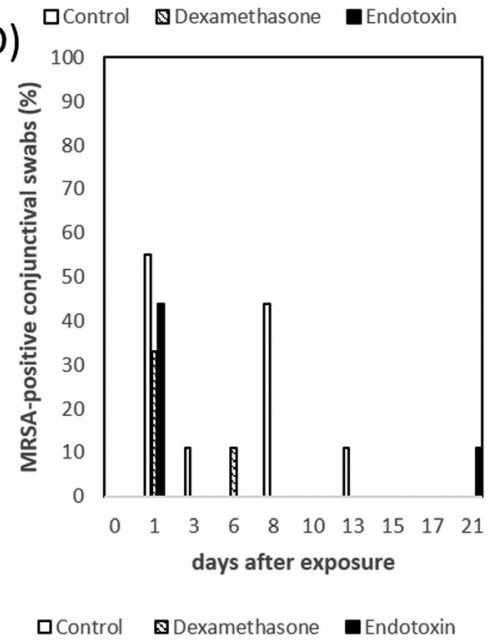

(B)

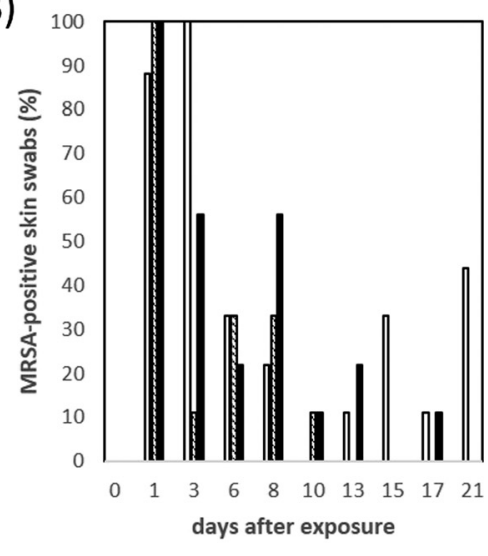

(E)
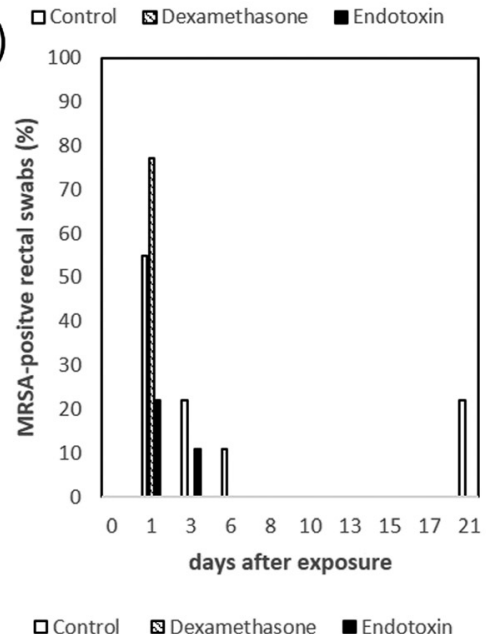

(C)

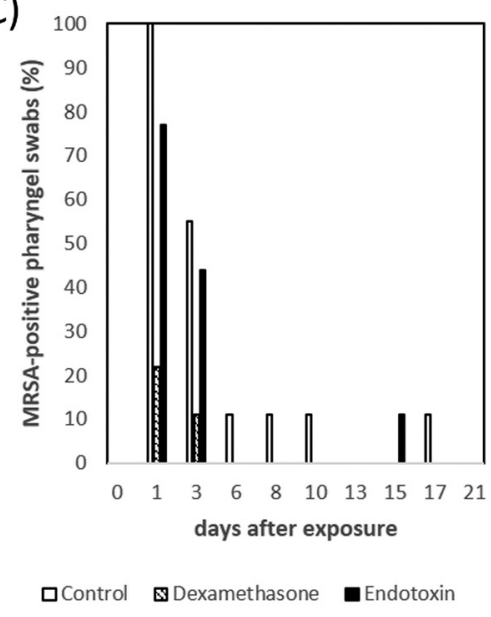

Figure 3. Percentages of MRSA-positive nasal (A), skin (B), pharyngeal (C), conjunctival (D), and rectal (E) swabs from the piglets of the control group (CG), dexamethasone group (DG) and endotoxin group (EG) group over the observation period

paired $t$-test) that was specific to the dexamethasone-treated group, while animals of the CG had unchanged total cell counts $(p=0.779)$. To assess immunosuppression in more detail, we performed differential cell counts and found that the decrease of total leucocytes was due to a specific decrease ( $p=0.001$, paired $t$-test) in numbers of lymphocytes, whereas the decrease in neutrophils was not significant $(p=0.392)$. However, the CG showed a significant increase $(p=0.002)$ in neutrophils and a significant decrease $(p=0.017)$ in lymphocyte counts. Therefore, we decided to more closely assess immunosuppression by dexamethasone treatment and evaluated the suppressive effects of the corticosteroid dexamethasone on lymphocytes in vitro. For that purpose, we stimulated PBMC labeled with the proliferation marker ConA, and assessed the frequencies of proliferated $\mathrm{CD} 4^{+} \mathrm{T}$ cell after 5 days by flow cytometry (Figure 4B). As shown in Figure $4 \mathrm{C}$ and $4 \mathrm{D}$, glucocorticoid treatment significantly reduced the capacity of $\mathrm{CD}^{+} \mathrm{T}$ cells to proliferate $(p=0.017$, paired $t$-test). In contrast, no significant effect on the proliferative capacity of $\mathrm{CD}^{+} \mathrm{T}$ cells was detected in the control group $(p=0.186)$.

Peripheral Blood Cell Counts. Figure 5A illustrates the mean total leukocyte counts over the entire experimental period for all groups. ANOVA analysis for repeated measurements revealed no significant differences in the leucocyte count over time between all groups ( $p=0.387 \mathrm{DG}$ versus $\mathrm{CG}$ and $p=0.466 \mathrm{EG}$ versus $\mathrm{CG}$ ).

Figure 5B depicts the neutrophil counts for all 3 groups during the observation period. In contrast to the DG $(p=0.867)$, statistical analysis revealed significant differences between the EG and the CG $(p=0.002)$ over time. The neutrophil counts of the EG were significantly increased compared to the CG from day -1 until the end of the observation period ( $p=\leq 0.001$ to $p=0.024$ ). The increase in neutrophil counts in the DG from day 1 to day 3 is notable, despite being not statistically significant compared to the other groups.

Figure 5C shows the lymphocyte counts for all 3 groups. The statistical analysis showed significant differences between all groups ( $p=0.004$ DG versus CG and $p=0.003$ EG versus $\mathrm{CG})$ over time.

\section{Discussion}

Our study aimed to investigate possible predisposing factors for MRSA colonization success focusing on the immunological state of the piglets. In addition to the stress induced by weaning and its effects on the adaptive immune system [15], piglets are exposed to new environmental conditions including climatic changes, different microorganisms, and dust containing bacterial endotoxins. Here, we imitated a weakened immunological state of weaned pigs via dexamethasone treatment prior to exposing them to MRSA, investigating the question if immune stress at this age promotes colonization by MRSA. Indeed, we hypothesized an extended MRSA colonization of dexamethasone-treated animals in contrast to animals exposed to airborne MRSA alone. However, our data show a significantly shorter MRSA colonization of dexamethasone-treated animals after an airborne exposure over time. Remarkably, most of the MRSA-positive samples of the DG were skin swabs showing a significant reduced detection compared to the CG indicating contamination rather than colonization. The poor ability of MRSA to colonize the piglets is 
(A)

Cell count of peripheral blood in the control group and dexamethasone grpup

(48 $\mathrm{h}$ effect of dexamethasone)

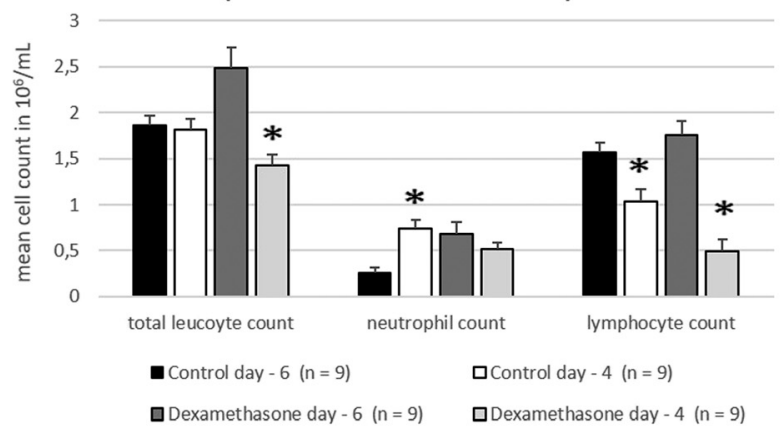

(B)

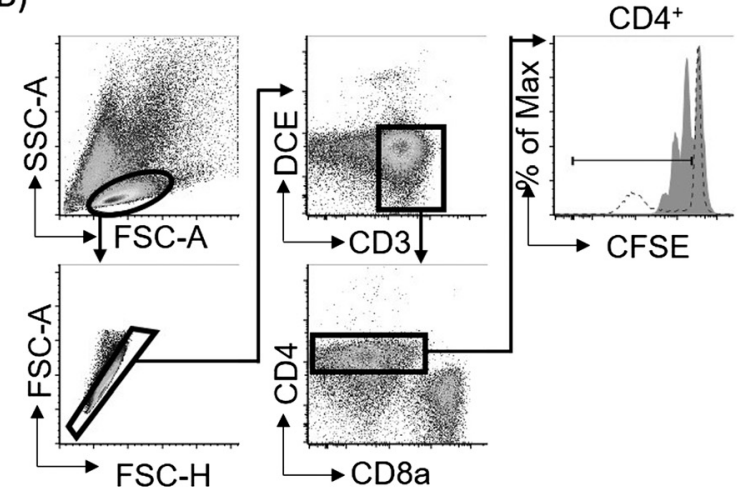

(C)

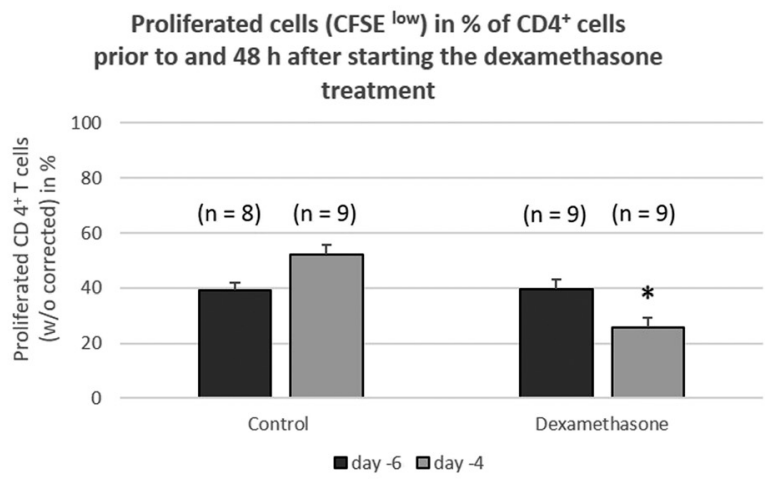

(D)

Proliferated cells (CFSE ${ }^{\text {low }}$ ) in \% of $\mathrm{CD4}^{+}$

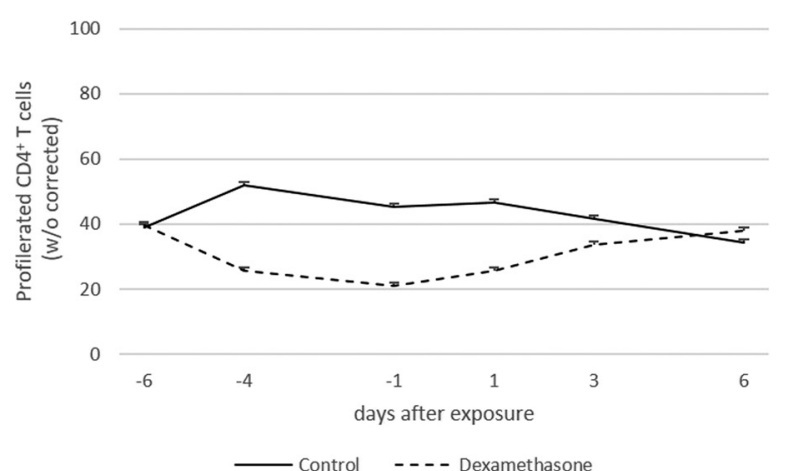

Figure 4. Dexamethasone treatment effects. A) The effect of dexamethasone administration (1 mg/kg body weight/day) on total leucocyte counts, neutrophil, and lymphocyte numbers at the beginning (day -6) and $48 \mathrm{~h}$ after the first application of Dexamethasone (day -4$)$. Asterisks indicate significantly different values comparing $t-6$ and $t-4: p \leq 0.005$. PBMCs were CFSE-labeled and stimulated with ConA (2 $\mu \mathrm{g} / \mathrm{mL})$ to assess their proliferative capacity. B) Flow cytometric gating strategy to analyze frequency of proliferated cells by $\mathrm{CFSE}-\mathrm{dilution}\left(\mathrm{CD} 3^{+} \mathrm{CD} 4{ }^{+} \mathrm{CFSE}{ }^{\text {low }}\right)$. C) Mean values of proliferated $\mathrm{CD}^{+}{ }^{\mathrm{T}}$ cells corrected for $\mathrm{CFSE}^{\text {low }}$ frequencies of unstimulated (w/o) controls. Asterisks indicate significant differences $(p=0.001)$ between $t-6$ (before treatment) and $t-4$ (after onset of Dexamethasone treatment). D) Mean values of proliferated CD4 ${ }^{+}$T cells corrected for $\mathrm{CFSE}^{\text {low }}$ frequencies of unstimulated (w/o) controls over prolonged observation time

presumably due to dexamethasone-induced higher MRSA clearance - an effect, which has not yet been investigated in piglets before. This is confirmed by the absence of MRSA in the nasal and rectal swabs from day 3 and the pharyngeal swabs from day 6 after exposure with a significantly lower number of MRSA-positive nasal swabs in the dexamethasonetreated animals compared to the CG. Although the sampling procedure was carried out with the greatest of care, the MRSA-positive conjunctival swab of 1 animal at day 3 might be a contamination due to its MRSA-positive skin at this sampling point.

The blood samples of our dexamethasone-treated piglets revealed a decrease of the lymphocyte count in peripheral blood in combination with decreased proliferation capacity of the $\mathrm{CD}^{+} \mathrm{T}$ cells, indicating mild immunomodulation of the adaptive immune system under dexamethasone application. A decline of lymphocytes after weaning was also observed in the study of Kick et al. and is a result of increased blood cortisol concentration induced by stress [15]. In prior studies, dexamethasone treatment also resulted in lymphocytopenia in pigs [8, 16-18]. Therefore, we assume that the dexamethasone treatment was partly effective to imitate the moderate stress occurring after weaning in the field; however, it is likely that there are other, uncharacterized effects of dexamethasone contributing to the defense of MRSA colonization. Additionally, other studies show that the success of immunosuppression by glucocorticoids in pigs is inconsistent, and Flaming et al. described pigs to be remarkably resistant to dexamethasone treatment with similar doses [18]. Our findings might be explained by different studies that investigated the effect of glucocorticoid treatment at low doses. In a recent review, Cain and Cidlowski summarized the effect of glucocorticoids on the immune system and proposed a biphasic, dose-dependent influence on rat models and human macrophages [19]. According to Lim et al. [20], a low-dose treatment of glucocorticoids resulted in an elevated expression of innate immune genes (for instance, cytokines and chemokines), sensitizing the organism towards pathogens. Therefore, a possible explanation for the decreased MRSA colonization in dexamethasone-treated animals is the stimulation of innate immunity by low-dose dexamethasone resulting in more rapid MRSA elimination. According to Kulkarni et al., dexamethasone treatment decreases the gene expression of antimicrobial peptides (AMPs) - secreted by innate immune cells (monocytes, macrophages, neutrophils and epithelial cells)-in THP-1 monocytes [21]. AMPs are involved in the early defense against pathogens and play, inter alia, a key role in the host cutaneous defense against $S$. aureus [22]. We hypothesize that AMP levels in the nasal epithelium of the piglets were reduced due to dexamethasone resulting in an enhanced adhesion ability of MRSA during the airborne MRSA exposure and its following microinvasion. The lower MRSA concentration (of free, non-attached or microinvaded MRSA) in the nasal swabs (DG) compared to the control directly after exposure might confirm this hypothesis. We assume a subsequent MRSA eradication by a strong local immune response and local inflammation attributed to the end of dexamethasone treatment at day 2 and its fast degradation [25]. This is 
Mean total cell count of peripheral blood cells in all three groups exposed to $10^{4} \mathrm{cfu} / \mathrm{m}^{3}$ MRSA in the air for 24 hours during the observation period

(A)

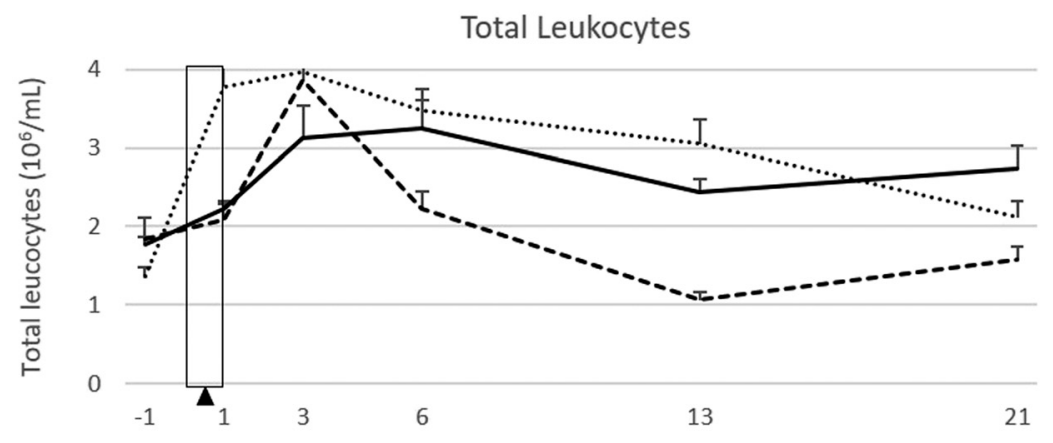

(B)

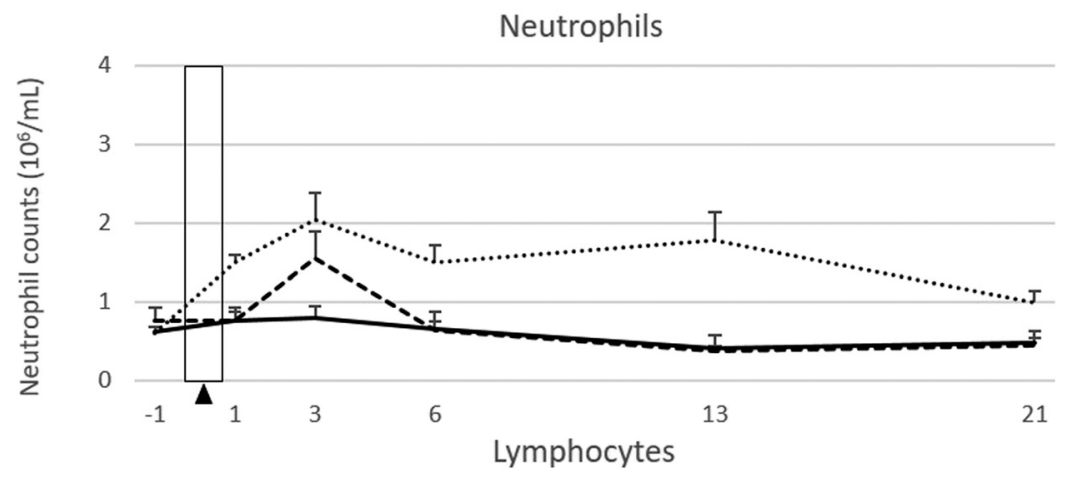

(C)

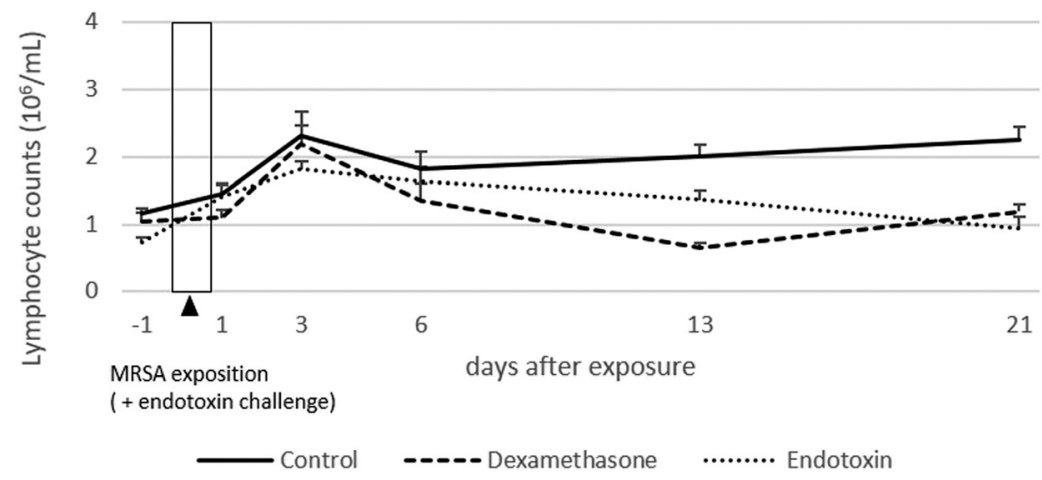

Figure 5. Mean total cell count of peripheral blood cells in the dexamethasone (DG), endotoxin (EG), and control group (CG) exposed to $10^{4} \mathrm{cfu} / \mathrm{m}^{3}$ MRSA in the air for $24 \mathrm{~h}$ during the observation period

supported by increased neutrophil counts in the peripheral blood at day 3, 1 day after stopping dexamethasone treatment. This hypothesis provides a further possible explanation for the significantly shorter MRSA colonization of the DG compared to the $\mathrm{CG}$, indicating low susceptibility to MRSA due to low-dose dexamethasone treatment combined with the specific time of stopping the treatment. According to our data, this treatment regimen does not promote MRSA colonization of piglets when exposed via the airborne transmission route.

We also examined the hypothesis that the presence of airborne endotoxin promotes airborne MRSA colonization by acting as a respiratory hazard. In our study, the piglets were exposed to an endotoxin-containing MRSA aerosol resulting in an airborne endotoxin concentration of $4 \mu \mathrm{g} / \mathrm{m}^{3}$, corresponding to the maximum endotoxin concentration found in pig barns [26]. However, the particle size of endotoxin is also a crucial factor influencing the place of deposition in the respiratory tract. In our study, the aerodynamic diameter of the aerosolized particles was between 3.2 and $3.7 \mu \mathrm{m}$ and endotoxin was, therefore, able to penetrate the alveolar region of the lung [27]. The statistical analyses demonstrate that there is no relationship between airborne endotoxin and MRSA colonization success in piglets, although our data reveal an endotoxin-associated effect on peripheral blood cells after endotoxin exposure. In humans, endotoxin is shown to damage respiratory endothelial cells [28] facilitating the ability of MRSA to colonize the respiratory mucosa. In light of possible injury to the upper respiratory tract epithelium, we expected a longer nasal and pharyngeal MRSA colonization acting as a source for spread and subsequent recolonization. Here again, the statistical analyses revealed no prolonged MRSA colonization in the EG compared to the CG, neither for the nose nor for the pharynx. The comparable nasal MRSA colonization of both groups suggests an intact nasal mucosa of the piglets despite airborne endotoxin exposure. Our data are supported by a study by Urbain et al. where no changes in the cell composition of nasal fluid after endotoxin nebulization were found, and therefore, the influence of airborne endotoxin on intact nasal mucosa was denied [10]. The cell counts of blood taken after exposure in the aerosol chamber (day 1) mirror the exposure to an endotoxin-containing MRSA aerosol and are not related to endotoxin alone. Since no changes in total leucocyte and neutrophil count were found after the exposure to airborne 
MRSA alone (CG), the increase of the total leukocytes due to a significant increase of neutrophils detected in the EG is interpreted as being induced by endotoxin. Alterations in the peripheral blood of endotoxin-exposed pigs were also found by other groups $[10,29]$. Thereby, our data imply that the effect of airborne endotoxin in our aerosol chamber on the immune system is comparable to those in animals continuously exposed to high levels of endotoxin in pig barns. Although airborne endotoxin exposure in the aerosol chamber resulted in a typical immune response, the effect on MRSA colonization success of piglets could not be found. One possible explanation might be the duration of endotoxin exposure. Shortterm exposure (i.e., $6 \mathrm{~h}$ ) had no effects on the composition of nasal lavage, bronchoalveolar lavage (BAL), and blood components attributed to the endotoxin exposure alone [31]. Jolie et al. found a significantly higher neutrophil and alveolar macrophage concentration in the BAL after long-term endotoxin exposure (i.e., 15 weeks), indicating a systemic inflammatory response, though in the absence of clinical signs [30]. Furthermore, in a field study, discrepancies were found in the immune response between pigs and broilers despite the equal endotoxin concentration in the environment of investigated barns [29]. Roque et al. explained their findings with the longer endotoxin exposure of pigs (5-month fattening period) compared to broilers (1-month fattening period) [29]. The authors concluded that endotoxin weakens the piglets' immune defense against pathogens underlining that a possible role of airborne endotoxin favoring MRSA colonization in field cannot be neglected. In livestock, pigs are constantly exposed to endotoxin during the complete fattening period and, therefore, a MRSA colonization promoting effect of endotoxin-containing air might be possible despite no effects being observed when exposing the animals for $24 \mathrm{~h}$ in an aerosol chamber.

Additional pollutants in the pigsties' environment (e.g., ammonia, carbon dioxide, and hydrogen sulfide) may also affect the airways and could influence, together with endotoxin, MRSA colonization as discussed by Urbain et al. [10]. The authors could show that exposing pigs to airborne endotoxin resulted in damaged nasal mucosa only when challenging the animals with ammonia beforehand. In another study by Folgemark et al., guinea pigs were exposed to endotoxin with and without $\beta(1,3)$-D-glucans - a cell wall component of fungiand found stronger inflammatory airway responses in the animals exposed to both agents compared to the animals challenged with endotoxin alone [32]. The findings of Urbain et al. and Folgemark et al. support the assumption that airborne endotoxin might be a contributing factor for developing respiratory diseases when combined with other common factors occurring in pig barn air $[10,32]$. Our results indicate that airborne endotoxin alone-at least when exposed for $24 \mathrm{~h}$ only-does not promote MRSA colonization in piglets.

The discrepancy between the airborne MRSA dose regularly found in pigsties $\left(10^{2} \mathrm{cfu} / \mathrm{m}^{3}\right)$ by Friese et al. [5] associated with the high MRSA prevalence in pigs and the effective dose for successful permanent colonization found in our animal trial $\left(10^{6} \mathrm{cfu} / \mathrm{m}^{3}\right.$ [4]) is presumably due to the multifactorial nature of the pigsties' environment. Pigs are naturally exposed to numerous other factors that may influence the ability of MRSA to colonize pigs. Apart from the diverse pollutants in the pigs' environment, the MRSA colonization might also be driven by treatment with antibiotics and metallic oxide. In our study, dexamethasone decreased the number of lymphocytes $48 \mathrm{~h}$ after treatment same as that of weaned piglets in the field [15]. Due to the understudied effects of dexamethasone, this treatment regime resulted in a higher clearance of MRSA and was, therefore, potentially not adequate to imitate chronic weaning stress in pigs occurring in intensive pig production. Airborne endotoxin exposure in the aerosol chamber did not lead to a different MRSA colonization. Hence, future studies should use combinations of possible predisposing factors promoting MRSA colonization in order to study successful MRSA colonization in pigs.

In conclusion, we have shown that the airborne LA-MRSA colonization is independent of the piglets' immune status. MRSA in the pigs' environment is considered one of the main sources for the animals' colonization [33, 34]. To prevent a reentry of dust-borne MRSA from particles deposited in the environment, reducing the MRSA burden by a stringent cleaning and disinfection regime is crucial.

\section{Funding Sources}

This study was funded in parts by the Federal Institute for Risk Assessment (BfR) with grant no. 1329-530.

\section{Authors' Contribution}

UR performed the study design. UR and AF designed the animal experiments. SH, FE, and SS performed the sampling and investigation protocol for the analysis of immunological parameters. FE and SS performed the immunological laboratory work and evaluated the dataset of the immunological parameters. RM performed the statistical analysis. KR performed the microbial laboratory work, performed the sampling, evaluated the final data, and wrote the manuscript. All authors have read and approved the final draft of the article.

\section{Conflict of Interest}

Nothing to declare.

\section{Supporting Information}

Number of MRSA-positive swab samples per animal of the 3 groups exposed to $10^{4} \mathrm{cfu} / \mathrm{m}^{3}$ MRSA in the air during the entire observation period. Each color represents 1 animal, which was sampled with 5 different swab samples for investigating the presence of MRSA, respectively.

Acknowledgements. The authors would like to thank Heike Rose, Susann Sellenthin, and Michael Kühl (Institute for Animal Hygiene and Environmental Health) for excellent technical assistance in the laboratory. A special thanks goes to the team of the animal keepers of the Centre for Infection Medicine, especially Sebastian Scheunemann and Sven Schäfer for the care of the animals and handling during sampling.

Futhermore, we thank the National Reference Laboratory for coagulase-positive staphylococci including Staphylococcus aureus and the Federal Institute for Risk Assessment [BfR] for providing the MRSA ST398 strain. We also acknowledge support by the German Research Foundation and the Open Access Publication Fund of the Freie Universität Berlin.

We also would like to thank Ankur Midha for proofreading this manuscript.

\section{References}

1. Vincze S, Stamm I, Kopp PA, Hermes J, Adlhoch C, Semmler T, Wieler LH, et al. Alarming proportions of methicillin-resistant Staphylococcus aureus (MRSA) in wound samples from companion animals, Germany 20102012. PloS one. 2014;9:e85656.

2. Cuny C, Witte W. MRSA in equine hospitals and its significance for infections in humans. Vet Microbiol. 2017;200:59-64.

3. Alt K, Fetsch A, Schroeter A, Guerra B, Hammerl JA, Hertwig S, Senkov N, et al. Factors associated with the occurrence of MRSA CC398 in herds of fattening pigs in Germany. BMC Vet Res. 2011;7:69.

4. Rosen K, Roesler U, Merle R, Friese A. Persistent and Transient Airborne MRSA Colonization of Piglets in a Newly Established Animal Model. Front Microbiol. 2018;9:1542. 
5. Friese A, Schulz J, Hoehle L, Fetsch A, Tenhagen B-A, Hartung J, Roesler U. Occurrence of MRSA in air and housing environment of pig barns. Vet Microbiol. 2012;158:129-35.

6. Amadori M, Stefanon B, Sgorlon S, Farinacci M. Immune system response to stress factors. Ital J Anim Sci. 2009;8:287-99.

7. Amadori $\mathrm{M}$, Zanotti $\mathrm{C}$. Immunoprophylaxis in intensive farming systems: The way forward. Vet Immunol Immunopathol. 2016;181:2-9.

8. Wallgren P, Wilén I-L, Fossum C. Influence of experimentally induced endogenous production of cortisol on the immune capacity in swine. Vet Immunol Immunopathol. 1994;42:301-16.

9. Holst H, Edqvist L-E, Kindahl H, Rylander R. Hematological, Blood Biochemical, and Cytological Bronchoalveolar Lavage Studies in Prepubertal Gilts after Endotoxin Inhalation and Ingestion. J Vet Med A. 1994;41:159-66.

10. Urbain B, Prouvost J-F, Beerens D, Ansay M, Gustin P. Acute Effects of Endotoxin Inhalation on the Respiratory Tract in Pigs: Interaction with Ammonia. Inhalation Toxicol. 1996;8:947-68.

11. Fogelmark B, Sjösstrand M, Rylander R. Pulmonary inflammation induced by repeated inhalations of f(1,3)-D-glucan and endotoxin. 1994:85-90. 12. Szabó I, Beck B, Friese A, Fetsch A, Tenhagen B-A, Roesler U. Colonization kinetics of different methicillin-resistant Staphylococcus aureus sequence types in pigs and host susceptibilities. Appl Environ Microbiol. 2012;78:541-48

13. Zejda JE, Barber E, Dosman JA, Olenchock SA, Mc Duffie HH, et al. Respiratory Health Status in Swine Producers Relates to Endotoxin Exposure in the Presence of Low Dust Levels. JOM. 1994;1994:49-56.

14. Kahl BC, Mellmann A, Deiwick S, Peters G, Harmsen D. Variation of the polymorphic region $\mathrm{X}$ of the protein $\mathrm{A}$ gene during persistent airway infection of cystic fibrosis patients reflects two independent mechanisms of genetic change in Staphylococcus aureus. J Clin Microbiol. 2005;43:502-5.

15. Kick AR, Tompkins MB, Flowers WL, Whisnant CS, Almond GW. Effects of stress associated with weaning on the adaptive immune system in pigs. J Anim Sci. 2012;90:649-56.

16. Harada K, Muramatsu M, Suzuki S, Tamura Y, Sawada T, Takahashi T. Evaluation on the pathogenicity of Erysipelothrix tonsillarum for pigs by immunosuppression with cyclophosphamide or dexamethasone. Res Vet Sci. 2011;90:20-2.

17. Salak-Johnson JL, McGlone JJ, Norman RL. In vivo glucocorticoid effects on porcine natural killer cell activity and circulating leucocytes. J Anim Sci. 1996;584-92.

18. Flaming KP, Goff BL, Frank DE, Roth JA. Pigs are Relatively Resistant to Dexamrthasone Induced Immunosuppression. Comp Haematol Int 1994;218-25.

19. Cain DW, Cidlowski JA. Immune regulation by glucocorticoids. Nat Rev Immunol. 2017;17:233-47.

20. Lim H-Y, Müller N, Herold MJ, van den Brandt J, Reichardt HM. Glucocorticoids exert opposing effects on macrophage function dependent on their concentration. Immunology. 2007;122:47-53.
21. Kulkarni NN, Gunnarsson HI, Yi Z, Gudmundsdottir S, Sigurjonsson OE, Agerberth B, Gudmundsson GH. Glucocorticoid dexamethasone downregulates basal and vitamin D3 induced cathelicidin expression in human monocytes and bronchial epithelial cell line. Immunobiology. 2016;221:24552 .

22. Ryu S, Song PI, Seo CH, Cheong H, Park Y. Colonization and infection of the skin by $S$. aureus: immune system evasion and the response to cationic antimicrobial peptides. Int J Mol Sci. 2014;15:8753-72.

23. Brown AF, Leech JM, Rogers TR, McLoughlin RM. Staphylococcus aureus Colonization: Modulation of Host Immune Response and Impact on Human Vaccine Design. Front Immunol. 2014;4:507.

24. Im S-A, Gerelchuluun T, Lee C-K. Evidence for Direct Inhibition of MHC-Restricted Antigen Processing by Dexamethasone. Immune network. 2014; 14:328-32.

25. Wyns H, Meyer E, Watteyn A, Plessers E, de Baere S, de Backer P, Croubels S. Pharmacokinetics of dexamethasone after intravenous and intramuscular administration in pigs. Vet J. 2013;198:286-88.

26. Zejda JE, Barber E, Dosman JA, Olenchock SA, McDuffie $\mathrm{HH}$ Rhodes C, Hurst T. Respiratory Health Status in Swine Producers Relates to Endotoxin Exposure in the Presence of Low Dust Levels. 1994;38:49-56.

27. Basinas I, Sigsgaard T, Kromhout H, Heederik D, Wouters IM Schlünssen V. A comprehensive review of levels and determinants of personal exposure to dust and endotoxin in livestock farming. J Exposure Sci Environ Epidemiol. 2015;25:123-37.

28. Thorn J. The inflammatory response in humans after inhalation of bacterial endotoxin: a review. Inflammation research official journal of the European Histamine Research Society. 2001;50:254-61.

29. Roque K, Shin KM, Jo JH, Lim GD, Song ES, Shin SJ, Gautam R, et al. Association between endotoxin levels in dust from indoor swine housing environments and the immune responses of pigs. J Vet Sci. 2018;19:331-38.

30. Jolie R, Bäckström L, Olsen L, Chase C. A 15-Week Eperimental Exposure of Pigs to Airborne Dust with Added Endotoxin in a Coontinious Flow Exposure Chamber. Can J Vet Res. 1999;129-37.

31. Urbain B, Mast J, Beerens D, N'guyen T, Goddeeris B, Ansay M, Gustin P. Effect of inhalation of dust and endotoxin on respiratory tracts of pigs. Am J Vet Res. 1999;60:1055-60.

32. Folgemark B, Sjöstrand M, Rylander R. Pulmonary inflammation induced by repeated inhalations of $\mathrm{f}(1,3)$-D-glucan and endotoxin. J Exp Path. 1994;85-90.

33. Pletinckx LJ, Verhegghe M, Dewulf J, Crombé F, de Bleecker Y, Rasschaert G, Goddeeris BM, de Man I. Screening of poultry-pig farms for methicillin-resistant Staphylococcus aureus: sampling methodology and within herd prevalence in broiler flocks and pigs. Infect Genet Evol. 2011;11:2133-37.

34. Broens EM, Espinosa-Gongora C, Graat EAM, Vendrig N, van der Wolf PJ, Guardabassi L, Butaye P, et al. Longitudinal study on transmission of MRSA CC398 within pig herds. BMC Vet Res. 2012;8:58. 